Marquette University

e-Publications@Marquette

$4-2019$

\title{
Charge-Transfer or Excimeric State? Exploring the Nature of The Excited State in Cofacially Arrayed Polyfluorene Derivatives
}

\author{
Ainur Abzhanova \\ Marquette University \\ Lena V. Ivanova \\ Marquette University \\ Denan Wang \\ Marquette University \\ Tushar S. Navale \\ Marquette University \\ Sameh H. Abdelwahed \\ Marquette University
}

See next page for additional authors

Follow this and additional works at: https://epublications.marquette.edu/chem_fac

Part of the Chemistry Commons

\section{Recommended Citation}

Abzhanova, Ainur; Ivanova, Lena V.; Wang, Denan; Navale, Tushar S.; Abdelwahed, Sameh H.; Ivanov, Maxim Vadimovich; Lindeman, Sergey V.; Rathore, Rajendra; and Reid, Scott A., "Charge-Transfer or Excimeric State? Exploring the Nature of The Excited State in Cofacially Arrayed Polyfluorene Derivatives" (2019). Chemistry Faculty Research and Publications. 997.

https://epublications.marquette.edu/chem_fac/997 


\section{Authors}

Ainur Abzhanova, Lena V. Ivanova, Denan Wang, Tushar S. Navale, Sameh H. Abdelwahed, Maxim Vadimovich Ivanov, Sergey V. Lindeman, Rajendra Rathore, and Scott A. Reid 
Marquette University

e-Publications@Marquette

\title{
Chemistry Faculty Research and Publications/College of Arts and Sciences
}

This paper is NOT THE PUBLISHED VERSION; but the author's final, peer-reviewed manuscript. The published version may be accessed by following the link in the citation below.

Journal of Photochemistry and Photobiology A : Chemistry, Vol. 371 (April 2019): 125-130. DOI. This article is (C) Elsevier and permission has been granted for this version to appear in $\underline{\text { e- }}$ Publications@Marquette. Elsevier does not grant permission for this article to be further copied/distributed or hosted elsewhere without the express permission from Elsevier.

\section{Charge-Transfer or Excimeric State? Exploring the Nature of The Excited State in Cofacially Arrayed Polyfluorene Derivatives}

\author{
Ainur Abzhanova \\ Marquette University \\ Lena V. Ivanova \\ Marquette University \\ Denan Wang \\ Marquette University \\ Tushar S. Navale \\ Marquette University
}

Sameh H. Abdelwahed

Marquette University

Maxim V. Ivanov

Marquette University

Sergey Lindeman

Department of Chemistry, Marquette University, Milwaukee, WI 


\section{Rajendra Rathore}

Department of Chemistry, Marquette University, Milwaukee, WI

Scott A. Reid

Department of Chemistry, Marquette University, Milwaukee, WI

\section{Highlights}

Nature of the excited state is probed in cofacially arrayed polyfluorene derivatives.

We examine whether an excimer to charge-transfer transition can be observed.

In all cases we find that excimer formation is energetically favorable.

Promotion of a charge-transfer state is possible but requires a free energy for electron transfer exceeding $1 \mathrm{~V}$.

These findings illuminate important design principles for molecular scaffolds capable of stabilizing both excimeric and charge-transfer states.

\section{Abstract}

It is well known that upon electronic excitation various $\pi$-stacked dimers readily exhibit excimer formation, facilitated by a perfect sandwich-like arrangement between the chromophores. However, it is unclear whether such a dimer is also capable of electron transfer upon excitation, if a strong electron-donating group is covalently attached. In this work, we probe the nature of the excited state in a series of cofacially arrayed polyfluorene derivatives with electron-rich aromatic donor attached via a methylene linker. Our studies show that in all cases excimer formation is energetically favorable, and promotion of a charge-transfer state in such systems is possible but requires a free energy for electron transfer far exceeding $1 \mathrm{~V}$. These findings shed light on important design principles for molecular scaffolds capable of stabilizing both excimeric and charge-transfer states upon their excitation.

\section{Keyword}

Charge transfer

\section{Introduction}

Understanding the nature of the excited state in $\pi$-stacked assemblies is critically important for development of efficient artificial light harvesting systems and photovoltaic devices [[1], [2], [3]]. It is now established that extensive exciton delocalization is a crucial factor in achieving efficient energy transfer in various molecular assemblies [4,5]. However, coupling to the internal and external vibrational modes leads to exciton localization, heavily restricting the size of exciton and reducing the rate of exciton migration [6,7]. In such a scenario it is assumed that exciton transfer follows an incoherent hopping mechanism, which allows several hops before emission [8].

As an example, photoexcitation of cofacially-arrayed polyfluorenes (Fn, Fig. 1A) leads to the formation of an excimeric state where exciton delocalization is limited to two fluorenes for all $n$, as evident from the characteristic band at $395 \mathrm{~nm}$ in the emission spectra of F2-F6 (Fig. 1B) [9,10]. This is understood as occurring due to the rapid decrease in stabilization with increasing $n$ in comparison with the geometrical penalty for reorganization into multiply stacked structures. Furthermore, we have recently shown that an identical band is observed in the emission spectrum of the rigid cyclophane-like bifluorene ${ }^{\mathrm{C}} \mathrm{F} 2$, providing further support that in the $\mathrm{F} n$ series exciton delocalization is limited to two fluorenes with a sandwich-like arrangement (Fig. 1C) [11]. 
A

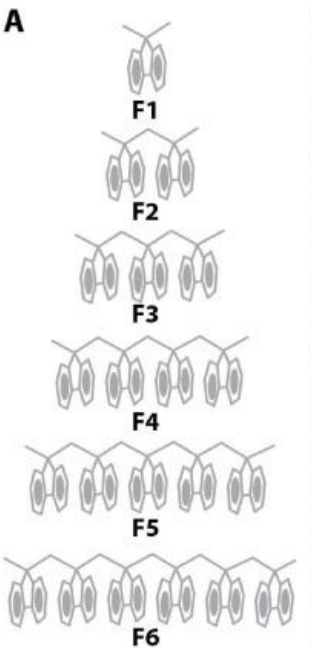

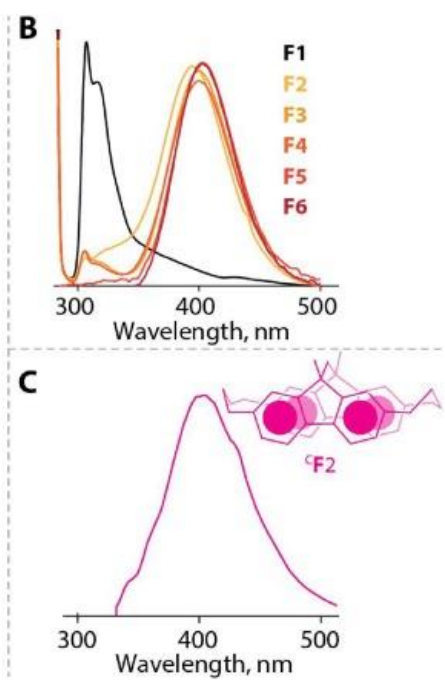

Fig. 1. A. Structures of cofacially-arrayed polyfluorenes $(\mathrm{F} n, n=1-6)$. B. Emission spectra of $\mathrm{F} 1-\mathrm{F} 6$ in $\mathrm{CH}_{2} \mathrm{Cl}_{2}$ at $22^{\circ} \mathrm{C}$. C. Emission spectrum of ${ }^{\mathrm{C}} \mathrm{F} 2 \mathrm{CH}_{2} \mathrm{Cl}_{2}$ at $22^{\circ} \mathrm{C}$.

While it is usually assumed that excimer formation acts as a trap, resulting in dissipation of the excitonic energy and concomitant loss of efficient charge separation, a recent study by Würthner and co-workers showed that the electron transfer step in covalently linked perylene bisimide dimers (PBI) occurs via formation of the excimeric state [12]. There, it was demonstrated that the decay of excimer emission is accompanied by the rise of transient bands assigned to the PBI cation and anion radicals. This observation calls for the studies to investigate the excited state dynamics in systems capable of stabilizing both excimeric and charge-transfer states.

Motivated by these findings, we synthesized a series of polyfluorene derivatives ( ${ }^{\mathrm{Ar}} \mathrm{F} n, n=1-3$, Chart 1 ) with an aromatic electron donor (i.e., $\mathrm{Ar}=2,5$-dimethoxy- $p$-xylene or $\mathrm{DMX}$ ) attached via a methylene linker. One may expect that upon excitation of ${ }^{A r} F n$, the aryl group would act as an electron donor ( $E_{\text {ox }}[\mathrm{DMX}]=1.12 \mathrm{~V}$ vs $\mathrm{SCE}$ ) and polyfluorene moiety as an electron acceptor $\left(E_{\text {ox }}[\mathbf{F} 1]=1.73 \mathrm{~V}\right.$ and $E_{\text {red }}[\mathbf{F} 1] \sim 2.2 \mathrm{~V}$ vs SCE$)$, thus favoring a chargetransfer state. On the other hand, accessibility of the sandwich-like arrangement in the polyfluorene moiety beginning from $n=2$ might favor stabilization of the excimeric state. In this manuscript, we probed the nature of the excited state in the Ar $\mathrm{F} n$ derivatives with the aid of steady-state optical spectroscopy and DFT calculations. We demonstrate that excimer formation is energetically favorable, and in order to promote the charge-transfer state in such systems, the free energy of electron transfer must far exceed $1 \mathrm{~V}$. Details of the preliminary findings on the nature of the excited state in ${ }^{\mathrm{Ar}} \mathrm{F} n$ are discussed below.

\section{Excimeric or charge transfer state?}

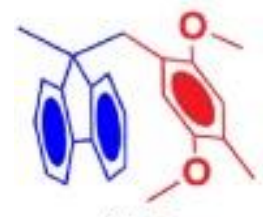

${ }^{\text {Ar }} \mathbf{F} 1$

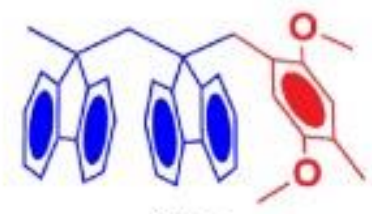

${ }^{A} \mathbf{F} 2$

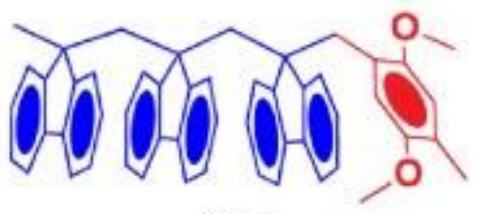

${ }^{A}$ F3

Chart 1. Structures of ( ${ }^{\mathrm{Ar}} \mathrm{F} n, n=1-3, \mathrm{Ar}=2,5$-dimethoxy- $p$-xylene). 


\section{Results and discussion}

\subsection{Synthesis}

A series of polyfluorene derivatives ${ }^{A r} F n(A r=2,5$-dimethoxy- $p$-xylene or $\mathrm{DMX}$, and $n=1-3$ ) were synthesized from readily available fluorene using the synthetic route illustrated in Scheme $1 \mathrm{~A}$. All compounds were characterized by ${ }^{1} \mathrm{H} /{ }^{13} \mathrm{C}$ NMR and MALDI-TOF mass spectroscopy. The structure of ${ }^{A r} \mathrm{~F} 3$ was further characterized by X-ray crystallography (Scheme 1B). Complete experimental details and characterization data are summarized in the Supporting Information.

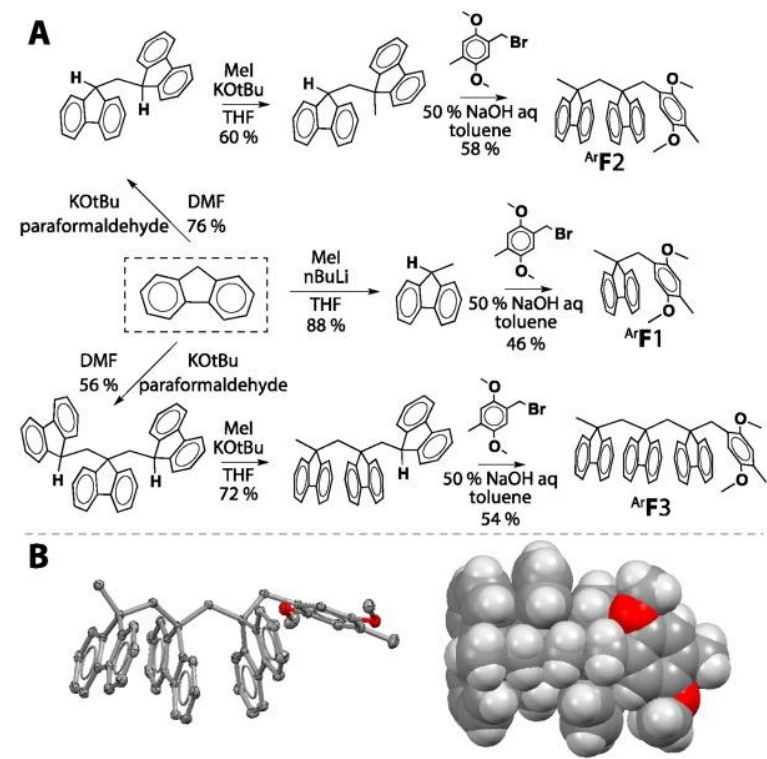

Scheme 1. A. Synthetic scheme of ${ }^{A r} F n(A r=2,5$-dimethoxy-p-xylene and $n=1-3)$. B. ORTEP diagram ( $50 \%$ probability) and space-filling representation of ${ }^{\mathrm{Ar}} \mathrm{F} n$.

\subsection{Electrochemistry of ${ }^{A r} F n$ and spectroscopy of their cation radicals}

The electron donor strengths of ${ }^{\mathrm{Ar}} \mathrm{F} n$ series ( $\mathrm{Ar}=2,5$-dimethoxy-p-xylene or $\mathrm{DMX}$, and $n=1-3$ ) were evaluated by electrochemical oxidation at a platinum electrode as a $2 \mathrm{mM}$ solution in $\mathrm{CH}_{2} \mathrm{Cl}_{2}$ containing $0.1 \mathrm{M} n-\mathrm{Bu}_{4} \mathrm{NPF}_{6}$ as the supporting electrolyte. Cyclic voltammograms (CVs) of ${ }^{\mathrm{Ar}} \mathrm{F} n$ showed one reversible oxidation wave in each case, with a minor lowering of the oxidation potential $\left(E_{o x}\right)$ upon increasing number of fluorenes (Fig. 2A). In contrast, unsubstituted polyfluorenes $(\mathrm{F} n)$ display a significant lowering of the $E_{\text {ox }}$ with increasing $n$, signifying extended delocalization of the cationic charge (i.e., hole) across the cofacially-arrayed polyfluorenes (Fig. 2A). As the $E_{\text {ox }}$ of ${ }^{A r} F n$ spans a narrow range of 0.66-0.63 $\mathrm{V}$ that includes $E_{\text {ox }}$ of $\mathrm{DMX}$, [13] the hole delocalization must be limited to the aryl moiety of ${ }^{A r} F n$, irrespective of the number of fluorenes. Indeed, calculations using CAMB3LYP-D3/6-31 G(d) [[14], [15], [16]] confirmed that: 1) the free energy of oxidation does not depend on the number of fluorenes, and 2) the hole is localized at the aryl moiety of ${ }^{A r} \mathrm{~F}^{+\bullet}$ as visualized using the isovalue spindensity plots in Fig. 2A. 

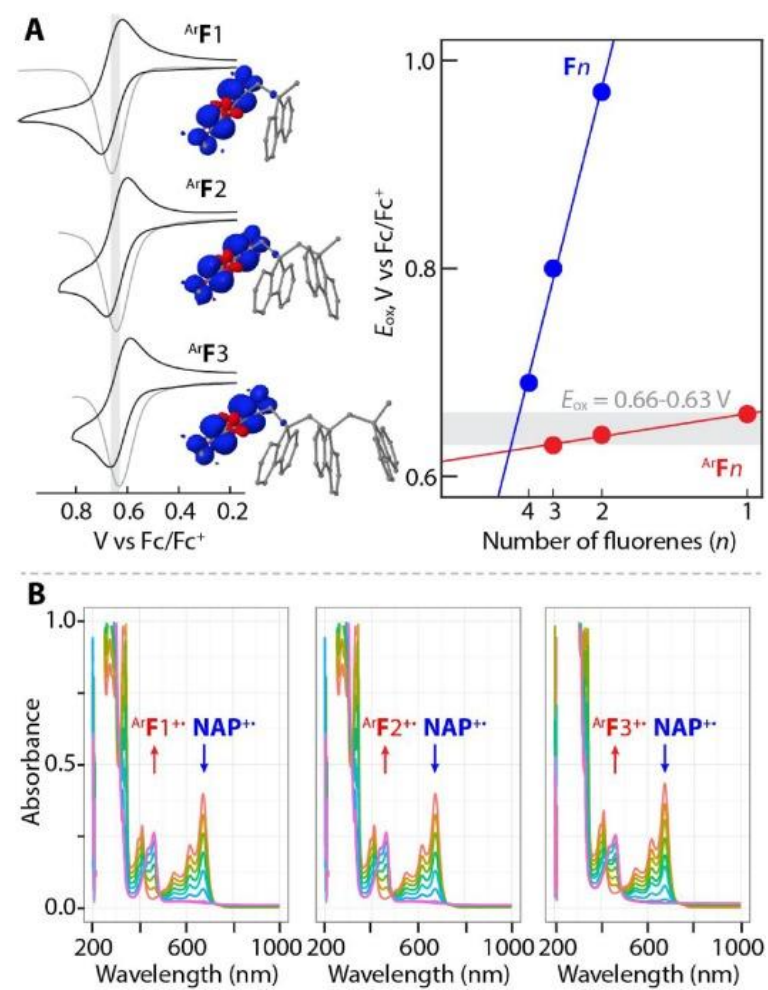

Fig. 2. Cyclic and square-wave voltammograms of $2 \mathrm{mM}{ }^{\mathrm{Ar}} \mathrm{F} n$ in $\mathrm{CH}_{2} \mathrm{Cl}_{2}$ at $22^{\circ} \mathrm{C}$; isovalue spin-density plots of ${ }^{\mathrm{Ar}} \mathrm{F}^{+\cdot}$ calculated using CAM-B3LYP-D3/6-31 G(d)+PCM $\left(\mathrm{CH}_{2} \mathrm{Cl}_{2}\right)$ and plots of experimental oxidation potentials of $\mathrm{F} n$ (blue) and ${ }^{A r}$ Fn (red) against $1 / n$.

The electrochemical stability of the ${ }^{\mathrm{Ar}} \mathrm{F} n$ series prompted us to generate their cation radicals using a stable cation-radical salt $\left[\mathrm{NAP}^{+}\right.$. $\mathrm{SbCl}_{6}{ }^{-}$] (NAP $=1,1,4,4,7,7,10,10$-octamethyl-1,2,3,4,7,8,9,10-

octahydrotetracene, $E_{\text {red }}=0.94 \mathrm{~V}$ vs Fc $/ \mathrm{Fc}^{+}, \lambda_{\max }=673 \mathrm{~nm}, \varepsilon_{\max }=9300 \mathrm{~cm}^{-1} \mathrm{M}^{-1}$ ) as a one-electron aromatic oxidant in $\mathrm{CH}_{2} \mathrm{Cl}_{2}$ [17]. The redox titration experiment was carried out by an incremental addition of substoichiometric amounts of ${ }^{A r} \mathrm{~F} n$ to the solution of $\mathrm{NAP}^{+}$. . The one-electron oxidation of ${ }^{\mathrm{Ar}} \mathrm{F} n$ to ${ }^{\mathrm{Ar}} \mathrm{F} n^{+}$. and reduction of $\mathrm{NAP}^{+}$. to NAP can be described by the equilibrium shown in Eq. (1).(1) NAP+. + ${ }^{\mathrm{Ar}} \mathrm{F} n \rightleftarrows{ }^{\mathrm{Ar}} \mathrm{F} n+{ }^{\mathrm{Ar}} \mathrm{F} n^{+}$.

Treatment of $\mathrm{NAP}^{+\bullet}$ with increments of ${ }^{\mathrm{Ar}} \mathrm{F} n$ led to disappearance of the absorption bands of $\mathrm{NAP}^{+}$. at $672 \mathrm{~nm}$ and growth of a new band at $468 \mathrm{~nm}$ (Fig. 3B). Numerical deconvolution $[18,19]$ of the absorption spectrum at each increment further confirmed that the redox reaction follows a 1:1 stoichiometry as indicated in Eq. (1). Comparison of the generated electronic spectra of ${ }^{\mathrm{Ar}} \mathrm{F}^{+}$. with that of model $\mathrm{DMX}^{+}$. confirmed that the sharp band at $468 \mathrm{~nm}$ arises due to the hole localization at the aryl moiety of ${ }^{\mathrm{Ar}} \mathrm{F} n^{+}$. in consistency with the electrochemical data and DFT calculations (Fig. 3A).
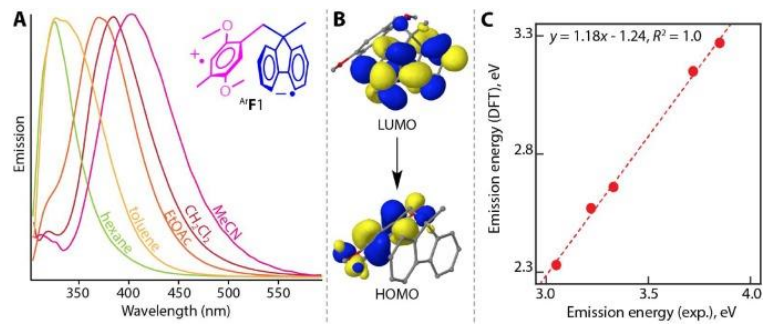

Fig. 3. A. Normalized emission spectra of ${ }^{A} F 1$ in various solvents. B. Isovalue plots of $H O M O$ and LUMO of ArF1 calculated using CAM-B3LYP-D3/6-31 G(d)+PCM $\left(\mathrm{CH}_{2} \mathrm{Cl}_{2}\right)$. C. Correlation plot between emission energies calculated using DFT and experimental energies of maximum emission of ${ }^{A r} \mathrm{~F} 1$ in various solvents. 
We emphasize that observation of a hole localization exclusively on the aryl group of ${ }^{A r} \mathrm{Fn}^{+}$. may seem unexpected, and further suggests that the electronic coupling between aryl group and adjacent fluorene is relatively minor. Yet, the electronic coupling in a structurally similar scaffold was found to be sufficient to promote the efficient energy transfer as has been shown in previous studies [20] of triplet energy transfer in the donor-bridge-acceptor system displayed in Scheme 2. In this study, the cofacially arrayed polyfluorenes acted as the bridge that was connected to a benzophenone triplet energy donor (Bp) and naphthalene acceptor (Nap) via the methylene linker. This study showed that the polyfluorenes act as an efficient medium for the energy transfer with the mechanism of the triplet energy transfer switching from tunneling to hopping when the number of fluorenes is changed from 1 to 2 .

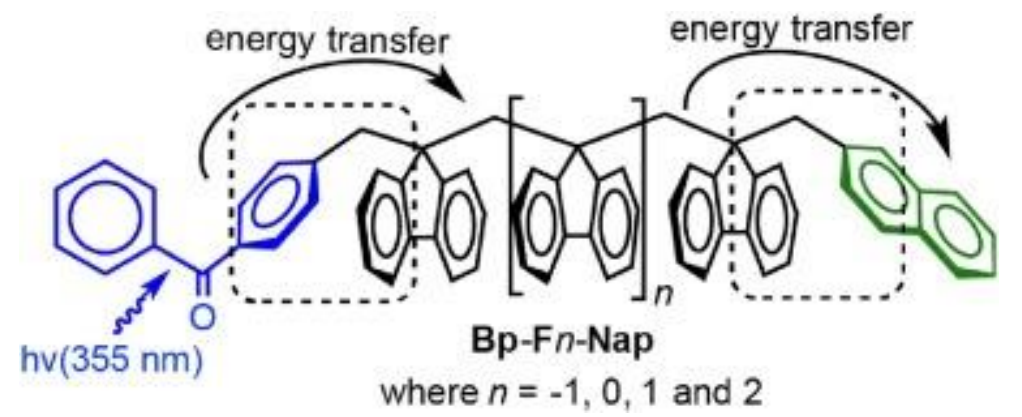

Scheme 2. Bp-Fn-Nap structures used to probe triplet energy transfer in cofacially arrayed polyfluorenes.

\subsection{Optical spectroscopy of ${ }^{A r}{ }_{F n}$}

To probe the nature of the (singlet) excited state in ${ }^{\mathrm{Ar}} \mathrm{F} n$ we recorded their absorption and emission spectra in solvents with varied polarity. Absorption spectra of ${ }^{A r} \mathrm{~F} n$ display characteristic bands with the wavelength of the absorption maximum invariant to the solvent polarity (Fig. S1 in the Supporting Information). In contrast, emission spectrum of Ar 1 show a strong dependence on solvent polarity, with the wavelength of the emission maximum evolving from $\lambda_{\mathrm{em}}=322 \mathrm{~nm}$ in hexane to $\lambda_{\mathrm{em}}=404 \mathrm{~nm}$ in acetonitrile (Fig. $3 \mathrm{~A}$ ).

Calculations using time-dependent (TD) DFT with CAM-B3LYP-D3/6-31 G(d) with state-specific solvation further confirm that emission of ${ }^{\mathrm{Ar}} \mathrm{F} 1$ at the equilibrium excited state geometry involves a transition between $\mathrm{HOMO}$ and LUMO, with the HOMO residing on the aryl group, and LUMO centered on the fluorene (Fig. 3B). Furthermore, emission energies calculated using TD-DFT showed a perfect linear correlation with the experimental values (Fig. 3C). Natural population analysis (NPA) [21] of the excited state wavefunction showed that a charge of $0.95 e$ is transferred upon excitation, which together with the experimentally observed strong solvent effect, clearly points to the charge-transfer nature of the excited state in ${ }^{A r} \mathbf{F} 1$.

In contrast, emission spectra of ${ }^{\mathrm{Ar}} \mathrm{F} 2$ and ${ }^{\mathrm{Ar}} \mathrm{F} 3$ derivatives show a characteristic excimeric band centered at $395 \mathrm{~nm}$ whose position is invariant to the solvent polarity (Fig. 4A and B). TD-DFT calculations confirmed that in ${ }^{A r}$ F2 exciton delocalization is spread over the two fluorenes, which form an ideal sandwich-like arrangement as represented in the transition density plots in Fig. 4C. Accordingly, excimeric emission involves transition from LUMO to HOMO, both centered on the same two-fluorene moieties. Finally, NPA of the excited state wavefunction in ${ }^{A r} F 2$ and ${ }^{A r} F 3$ showed that only a negligible amount of charge (i.e., $0.04 e$ ) is transferred to the bifluorene moiety upon excitation. 

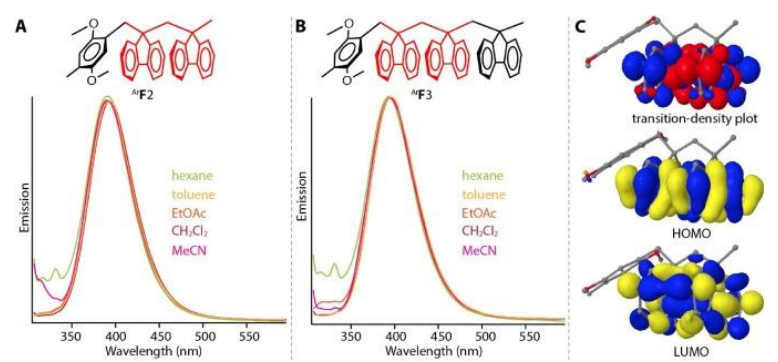

Fig. 4. Emission spectra of (A) ${ }^{A r} F 2$ and (B) ${ }^{A r} F 3$ in various solvents. C. Isovalue plots of transition density, HOMO and LUMO calculated using CAM-B3LYP-D3/6-31 G(d)+PCM $\left(\mathrm{CH}_{2} \mathrm{Cl}_{2}\right)$.

The striking transition in nature of the excited state from being purely charge-transfer in ${ }^{\mathrm{Ar}} \mathbf{F} 1$ to the excimeric in ${ }^{A} \mathrm{~F} n$ when $n=2-3$ provides important information on the stability of excimer formation in polyfluorenes. In particular, comparison of the redox potentials of the $\operatorname{Ar}$ group ( $E_{\text {ox }}[\mathrm{DMX}]=1.12 \mathrm{~V}$ vs SCE) and fluorene moiety $\left(E_{\mathrm{red}}[\mathrm{F} 1] \sim 2.2 \mathrm{~V}\right.$ vs SCE$)$ provides an estimated free energy of the electron transfer, i.e., $\Delta G_{\mathrm{ET}}=E_{\mathrm{ox}}[\mathrm{DMX}]-E_{\mathrm{red}}[\mathbf{F} 1]$ $=-1.1 \mathrm{~V}$. Thus, a significant energy gain in the electron transfer is responsible for the observed charge transfer upon excitation of ${ }^{A r} \mathbf{F} 1$. In contrast, while the presence of additional fluorenes in ${ }^{A r} \mathrm{~F} 2$ and ${ }^{A r} \mathrm{~F} 3$ is expected to further stabilize the anionic charge [22] and favor charge-transfer, the observation of an excimeric state in ${ }^{A r}$ F 2 and ArF3 suggests that the stabilization energy of the excimer exceeds that of the energetic gain of charge transfer $\left(\Delta G_{\mathrm{ET}}\right)$. These preliminary findings show that energy of excimer formation is significant and a much larger difference in the redox properties between the Ar group and polyfluorene moiety is needed in order to promote the charge transfer in ${ }^{A r} \mathrm{~F} n$ scaffolds. The preparation of such systems is currently under investigation and will be presented in due course.

\section{Conclusions}

Motivated by the recent reports on the stabilization of the electron-transfer state via excimer formation in perylene bisimide dimers (PBI), we explored the nature of the excited state in a series of cofacially arrayed polyfluorene derivatives ( ${ }^{\mathrm{Ar}} \mathrm{F} n$ ) with an electron-rich aromatic donor attached via methylene linker. Our preliminary studies showed that ${ }^{A r} \mathrm{~F} 1$ exhibits a charge-transfer state upon excitation, as evidenced in the strong dependence of the emission on the solvent polarity and supported by DFT calculations. In contrast, emission spectra of ${ }^{A r} \mathrm{~F} 2$ and ${ }^{A r} \mathrm{~F} 3$ show a characteristic excimeric band without any solvent effect, suggesting that an excimeric state is formed, an observation also supported by DFT calculations. The transition of the nature of the excited state from charge-transfer like in ${ }^{A r} \mathrm{~F} 1$ to excimer like in ${ }^{A r} \mathrm{~F} 2$ and ${ }^{A r} \mathrm{~F} 3$ suggests a significant energetic gain associated with excimer formation. Finally, comparison of the redox potentials of aromatic group and fluorene moiety suggest that the free energy of the electron transfer must exceed $1.1 \mathrm{~V}$ in order to favor formation of the charge-transfer state in polyfluorene scaffolds. These findings provide important guidance into the design and synthesis of molecular scaffolds capable of stabilizing both excimeric and charge-transfer states upon their excitation.

\section{References}

[1] G.D. Scholes, G.R. Fleming, A. Olaya-Castro, R. van Grondelle. Lessons from nature about solar light harvesting. Nat. Chem., 3 (2011), pp. 763-774

[2] G.D. Scholes, G. Rumbles. Excitons in nanoscale systems. Nat. Mater., 5 (2006), pp. 683-696

[3] K.H. Park, W. Kim, J. Yang, D. Kim. Excited-state structural relaxation and exciton delocalization dynamics in linear and cyclic $\pi$-conjugated oligothiophenes. Chem. Soc. Rev., 47 (2018), pp. 4279-4294

[4] I. Kassal, J. Yuen-Zhou, S. Rahimi-Keshari. Does coherence enhance transport in photosynthesis? J. Phys. Chem. Lett., 4 (2013), pp. 362-367 
[5] F. Fassioli, R. Dinshaw, P.C. Arpin, G.D. Scholes. Photosynthetic light harvesting: excitons and coherence. J. R. Soc. Interface, 11 (2014) 20130901

[6]A. Schubert, V. Settels, W. Liu, F. Würthner, C. Meier, R.F. Fink, S. Schindlbeck, S. Lochbrunner, B. Engels, V. En gel. Ultrafast exciton self-trapping upon geometry deformation in perylene-based molecular aggregates. J. Phys. Chem. Lett., 4 (2013), pp. 792-796

[7] N.J. Hestand, F.C. Spano. Expanded theory of H-and J-Molecular aggregates: the effects of vibronic coupling and intermolecular charge transfer. Chem. Rev., 118 (2018), pp. 7069-7163

[8] J. Sung, P. Kim, B. Fimmel, F. Würthner, D. Kim. Direct observation of ultrafast coherent exciton dynamics in helical $\pi$-stacks of self-assembled perylene bisimides. Nat. Commun., 6 (2015), p. 8646

[9] R. Rathore, S.H. Abdelwahed, I.A. Guzei. Synthesis, structure, and evaluation of the effect of multiple stacking on the electron-donor properties of pi-stacked polyfluorenes. J. Am. Chem. Soc., 125 (2003), pp. 8712-8713

[10] M.R. Talipov, M.V. Ivanov, S.A. Reid, R. Rathore. Two's company, three's a crowd: exciton localization in cofacially arrayed polyfluorenes. J. Phys. Chem. Lett., 7 (2016), pp. 2915-2920

[11] W. Denan, M.M. Ivanov, K. Damian, L. John, C. Jin-Zhe, S.S. Reid, R. Rajendra. The role of torsional dynamics on hole and exciton stabilization in Ï-Stacked assemblies: design of rigid torsionomers of a cofacial bifluorene. Angew. Chem. Int. Ed., 57 (2019), pp. 8189-8193

[12] J. Sung, A. Nowak-Król, F. Schlosser, B. Fimmel, W. Kim, D. Kim, F. Würthner. Direct observation of excimermediated intramolecular Electron transfer in a cofacially-stacked perylene bisimide pair. J. Am. Chem. Soc., 138 (2016), pp. 9029-9032

[13] T.S. Navale, M.R. Talipov, R. Shukla, R. Rathore. Interplay between entropy and enthalpy in (Intramolecular) cyclophane-like folding versus (Intermolecular) dimerization of diarylalkane cation radicals. J. Phys. Chem. C, 120 (2016), pp. 19558-19565

[14] T. Yanai, D.P. Tew, N.C. Handy. A new hybrid exchange-correlation functional using the Coulombattenuating method (CAM-B3LYP). Chem. Phys. Lett., 393 (2004), pp. 51-57

[15] S. Grimme, J. Antony, S. Ehrlich, H. Krieg. A consistent and accurate ab initio parametrization of density functional dispersion correction (DFT-D) for the 94 elements H-Pu. J. Chem. Phys., 132 (2010) 154104

[16] W.J. Hehre, R. Ditchfield, J.A. Pople. Selfconsistent molecular orbital methods. XII. Further extensions of gaussiantype basis sets for use in molecular orbital studies of organic molecules. J. Chem. Phys., 56 (1972), pp. 2257-2261

[17] M.R. Talipov, R. Rathore. Robust aromatic cation radicals as redox tunable oxidants. Organic Redox Systems: Synthesis, Properties, and Applications, John Wiley \& Sons, Hoboken, NJ (2015), p. 131

[18] M.R. Talipov, A. Boddeda, M.M. Hossain, R. Rathore. Quantitative generation of cation radicals and dications using aromatic oxidants: effect of added electrolyte on the redox potentials of aromatic electron donors. J. Phys. Org. Chem., 29 (2015), pp. 227-233

[19] M.R. Talipov, M.M. Hossain, A. Boddeda, K. Thakur, R. Rathore. A search for blues brothers: X-ray crystallographic/spectroscopic characterization of the tetraarylbenzidine cation radical as a product of aging of solid magic blue. Org. Biomol. Chem., 14 (2016), pp. 2961-2968

[20] J. Vura-Weis, S.H. Abdelwahed, R. Shukla, R. Rathore, M.A. Ratner, M.R. Wasielewski. Crossover from single-step tunneling to multistep hopping for molecular triplet energy transfer Science., 328 (2010), pp. 1547-1550

[21] A.E. Reed, R.B. Weinstock, F. Weinhold. Natural population analysis. J. Chem. Phys., 83 (1985), pp. 735-746

[22] R. Rathore, S.H. Abdelwahed, M.K. Kiesewetter, R.C. Reiter, C.D. Stevenson. Intramolecular electron transfer in cofacially pi-stacked fluorenes: evidence of tunneling. J. Phys. Chem. B, 110 (2006), pp. $1536-1540$ 